\title{
The Number of Different Distances Determined by a Set of Points in the Euclidean Plane*
}

\author{
Fan R. K. Chung, ${ }^{1}$ E. Szemerédi, ${ }^{2}$ and W. T. Trotter ${ }^{3}$ \\ ${ }^{1}$ Bell Communications Research, 445 South Street, Morristown, NJ 07962, USA \\ ${ }^{2}$ Mathematics Institute of the Hungarian Academy of Sciences, \\ Budapest, Hungary, and \\ Department of Computer Science, Rutgers University, \\ New Brunswick, NJ 08903, USA \\ ${ }^{3}$ Bell Communications Research, 445 South Street, \\ Morristown, NJ 07962, USA, and \\ Department of Mathematics, Arizona State University, \\ Tempe, AZ 85287, USA
}

\begin{abstract}
In $1946 \mathrm{P}$. Erdös posed the problem of determining the minimum number $d(n)$ of different distances determined by a set of $n$ points in the Euclidean plane. Erdös proved $d(n) \geq c n^{1 / 2}$ and conjectured that $d(n) \geq c n / \sqrt{\log n}$. If true, this inequality is best possible as is shown by the lattice points in the plane. We show $d(n) \geq n^{4 / 5} /(\log n)^{c}$.
\end{abstract}

\section{Introduction}

In 1946 Erdös [3] posed the problem of determining the minimum number $d(n)$ of different distances determined by a set of $n$ points in the Euclidean plane. Erdös proved $d(n) \geq c n^{1 / 2}$ and conjectured that $d(n) \geq c n / \sqrt{\log n}$. If true, this inequality is best possible as is shown by the lattice points in the plane.

Despite its apparent simplicity, progress on this problem has been slow. In 1952 Moser [4] proved that $d(n) \geq c n^{2 / 3}$ and this result stood as the best-known lower bound on $d(n)$ for more than 30 years. In 1984 Chung [2] proved $d(n) \geq c n^{5 / 7}$. Subsequently Beck [1] improved this to $d(n) \geq n^{(58 / 81)-\varepsilon}$.

Using the methods introduced by Szemerédi and Trotter [6], [7] on incidences

* The research of W. T. Trotter was supported in part by the National Science Foundation under DMS 8713994 and DMS 89-02481. 
between points and lines, and by Spencer, Szemerédi, and Trotter [5] on unit distances, it is relatively straightforward to show that $d(n) \geq c n^{3 / 4}$. However, in this article we combine techniques to show that $d(n) \geq n^{4 / 5} /(\log n)^{c}$.

Throughout this paper we consider finite sets of points in the Euclidean plane. We impose a cartesian coordinate system and choose a unit distance. When $\boldsymbol{x}=\left(x_{1}, x_{2}\right)$ and $\boldsymbol{y}=\left(y_{1}, y_{2}\right)$ are points in the plane, we let dist $(\boldsymbol{x}, \boldsymbol{y})$ denote the distance between $\boldsymbol{x}$ and $\boldsymbol{y}$, i.e., $\operatorname{dist}(\boldsymbol{x}, \boldsymbol{y})=\sqrt{\left(x_{1}-y_{1}\right)^{2}+\left(x_{2}-y_{2}\right)^{2}}$.

When $\boldsymbol{P}=\left\{\boldsymbol{x}_{1}, \boldsymbol{x}_{2}, \ldots, \boldsymbol{x}_{n}\right)$ is a set of $n$ distinct points in the plane, we let $\boldsymbol{D}(\boldsymbol{P})$ denote the set of distances determined by the points in $\boldsymbol{P}$, i.e., $\boldsymbol{D}(\boldsymbol{P})=\left\{\operatorname{dist}\left(\boldsymbol{x}_{i}, \boldsymbol{x}_{j}\right)\right.$ : $1 \leq i<j \leq n\}$. We also let $\boldsymbol{d}(\boldsymbol{P})=|\boldsymbol{D}(\boldsymbol{P})|$ so that $\boldsymbol{d}(\boldsymbol{P})$ counts the number of different distances determined by $\boldsymbol{P}$. Using this notation, the principal goal of this paper is to prove the following theorem.

Theorem 1. There exists an absolute constant $c_{1}>0$ so that if $n \geq 10$ and $P$ is any set of $n$ distinct points in the Euclidean plane, then the number $\boldsymbol{d}(\boldsymbol{P})$ of different distances determined by $\boldsymbol{P}$ satisfies $\boldsymbol{d}(\boldsymbol{P})>n^{4 / 5} /(\log n)^{c_{1}}$.

Our proof for Theorem 1 depends in part on the following inequality of Szemerédi and Trotter [7].

Theorem 2. There exists an absolute constant $c_{2}>0$ so that if $2 \leq k \leq \sqrt{n}$ and $\boldsymbol{P}$ is a set of $n$ points in the plane, then the number of lines containing at least $k$ points of $\boldsymbol{P}$ is less than $c_{2} n^{2} / k^{3}$.

\section{Distances-The Bipartite Version}

In order to establish Theorem 1, we first prove a bipartite version from which Theorem 1 follows as an immediate corollary. When $P$ and $Q$ are disjoint sets of points in the plane, we let $D(P, Q)=\{\operatorname{dist}(x, y): x \in P, y \in Q\}$ and $d(P, Q)=$ $|\boldsymbol{D}(\boldsymbol{P}, \boldsymbol{Q})|$.

Theorem 3. There exists an absolute constant $c_{3}>0$ so that if $n \geq 10$, and $\boldsymbol{P}$ and $\boldsymbol{Q}$ are disjoint point sets in the Euclidean plane with $|\boldsymbol{P}|=|\boldsymbol{Q}|=n$, then $d(\boldsymbol{P}, \boldsymbol{Q})>$ $n^{4 / 5} /(\log n)^{c_{3}}$.

Proof. We will show that the desired inequality holds when $c=c_{3}$ is sufficiently large. Our argument requires that $c$ be suitably large in comparison with the absolute constant $c_{2}$ in Theorem 2 . Although it is widely believed that Theorem 2 holds for some $c_{2}$ less than 10, the proof given in [7] yields the value $c_{2}=10^{180}$. So in the remainder of the proof, we take $c=c_{3}=10^{200}$, admittedly a generous choice.

Now suppose that the conclusion of the theorem fails for this value of $c$. Choose the least value of $n$ for which this occurs and let $P$ and $Q$ be disjoint $n$-element point sets in the plane for which $d(P, Q) \leq n^{4 / 5}(\log n)^{c}$. 
For each $i=0,1,2, \ldots, 20$, let $M_{i}=(\log n)^{10 i}$ and let $\left\lceil n / M_{i}\right\rceil$. Note that $n_{0}=n$. In what follows, we identify subsets of $\boldsymbol{P}$ and $\boldsymbol{Q}$ which will satisfy certain desired properties. This is done in a series of (at most 20) steps, and at each step we select a subset of the set resulting from the previous step. The sizes of the sets behave like the sequence $n_{0}, n_{1}, \ldots, n_{20}$. The reader may note that the argument only requires that $n_{i+1}$ be a small positive fraction of $n_{i}$, except at one critical step where we actually require that $n_{i+1}$ be smaller than $n_{i}$ by a logarithmic factor. However, the presentation of the argument is simplified by using the most restrictive requirement at each step.

Of all the discs which cover at least $n_{1}$ points of $P$, choose one, say $D_{1}$, so that the radius $r_{1}$ of $D_{1}$ is as small as possible. We consider points on the circular boundary of the disc to be covered by the disc. Also, choose a disc $D_{2}$ of minimum radius $r_{2}$ covering at least $n_{1}$ points of $Q$. We assume $r_{1} \leq r_{2}$; else reverse the roles played by $\boldsymbol{P}$ and $\boldsymbol{Q}$ in the remainder of the argument. Let $\boldsymbol{u}_{0}$ denote the center of disc $D_{1}$, and choose a subset $\boldsymbol{P}_{1}$ consisting of $n_{1}$ points of $\boldsymbol{P}$ covered by $D_{1}$.

Claim. There exists a number $r_{0}$ satisfying $r_{0} \geq 10^{10} r_{1}$ for which there are at least $10^{20} n_{1}$ points of $\boldsymbol{Q}$ in the annulus $\left\{\boldsymbol{y} \in \mathbf{R}^{2}: r_{0} \leq \operatorname{dist}\left(\boldsymbol{u}_{0}, \boldsymbol{y}\right) \leq r_{0}+3 r_{1}\right\}$.

Proof. Suppose the claim is false. Set $K=\left\lceil n / 10^{40} n_{1}\right\rceil$, and define a sequence $s_{0}$, $s_{1}, \ldots, s_{2 K-1}$ and pairwise disjoint subsets $S_{0}, S_{1}, S_{2}, \ldots, S_{2 K-1}$ of $Q$ as follows. First, set $s_{0}=10^{10} r_{1}$ and $S_{0}=\left\{\boldsymbol{y} \in Q: 0 \leq \operatorname{dist}\left(\boldsymbol{u}_{0}, \boldsymbol{y}\right) \leq s_{0}\right\}$. It is easy to see that $S_{0}$ can be covered by $10^{25}$ circles of radius $r_{1} / 2$, so we can safely say $\left|S_{0}\right|<10^{30} n_{1}$.

When $i$ is even, let $s_{i+1}$ be the least number exceeding $s_{i}$ for which the set $\boldsymbol{S}_{i+1}=\left\{\boldsymbol{y} \in \boldsymbol{Q}: s_{i} \leq \operatorname{dist}\left(\boldsymbol{u}_{0}, \boldsymbol{y}\right) \leq s_{i+1}\right\}$ contains at least $10^{20} n_{1}$ points. Since $\boldsymbol{d}(\boldsymbol{P}, \boldsymbol{Q})=d<n_{1}$, we can safely say $10^{20} n_{1} \leq\left|S_{i+1}\right| \leq 10^{30} n_{1}$ when $i$ is even. The upper bound follows from the fact that it is impossible for any circle centered at $\boldsymbol{u}_{0}$ to contain $2 n_{1}$ points of $\boldsymbol{Q}$, for this would imply that any point of $\boldsymbol{P}$ not located at $\boldsymbol{u}_{0}$ determines $n_{1}$ different distances with these points.

When $i$ is odd, we take $s_{i+1}=s_{i}+3 r_{1}$ and

$$
S_{i+1}=\left\{y \in Q: s_{i}<\operatorname{dist}\left(u_{0}, y\right)<s_{i+1}\right\}
$$

By assumption, $\left|S_{i+1}\right|<10^{30} n_{1}$. Now consider the pairwise disjoint subsets $S_{1}, S_{3}, S_{5}, \ldots, S_{2 K-1}$. Each contains more than $n_{1}$ points so that

$$
d\left(P_{1}, S_{2 j-1}\right)>n_{1}^{4 / 5} /\left(\log n_{1}\right)^{c}
$$

for each $j=1,2, \ldots, K$. Furthermore, if $\boldsymbol{x} \in \boldsymbol{P}_{1}$ and $\boldsymbol{y} \in \boldsymbol{S}_{2 j-1}$, then

$$
s_{2 j-2}-r_{1} \leq \operatorname{dist}(\boldsymbol{x}, \boldsymbol{y}) \leq s_{2 j-1}+r_{1}
$$


If $1 \leq j<k \leq K$, then $s_{2 j-1}+3 r_{1} \leq s_{2 k-2}$, so $s_{2 j-1}+r_{1} \leq\left(s_{2 k-2}-r_{1}\right)-r_{1}$, i.e., all distances in $D\left(P_{1}, S_{2 j-1}\right)$ are smaller than all distances in $D\left(P_{1}, S_{2 k-1}\right)$. Thus

$$
\begin{aligned}
d(P, Q) & \geq \frac{K n_{1}^{4 / 5}}{\left(\log n_{1}\right)^{c}} \\
& >\frac{\left\lceil n / 10^{40} n_{1}\right\rceil n_{1}^{4 / 5}}{(\log n)^{c}} \\
& \geq \frac{\left((\log n)^{2} / 10^{40}\right) n^{4 / 5}}{(\log n)^{c}}
\end{aligned}
$$

Since $n^{4 / 5} /(\log n)^{c}>d(P, Q)$, it follows that $\log n \leq 10^{20}$. To obtain a contradiction and complete the proof of the claim, we employ Erdös' original argument [3]. Choose distinct points $\boldsymbol{x}, \boldsymbol{x}^{\prime} \in \boldsymbol{P}$. If there exists a circle $C$ centered at $\boldsymbol{x}$ containing a subset $Q(C)$ of at least $\sqrt{n}$ points of $Q$, then $d\left(\left\{x^{\prime}\right\}, Q(C)\right) \geq|Q(C)| / 2 \geq$ $\sqrt{n} / 2$. Thus $n^{4 / 5} /(\log n)^{c} \geq \sqrt{n} / 2$, which fails when $(\log n) \leq 10^{20}$ which is forced by the size of the constant $c$. However, if no such circle exists, then $d(P, Q) \geq$ $d(\{x\}, Q) \geq \sqrt{n}$, which cannot hold when $(\log n) \leq 10^{20}$. The contradiction completes the proof of the claim.

By the pigeon-hole principle, we can choose two rays emanating from $\boldsymbol{u}_{0}$ and enclosing an angle of $5^{\circ}$ for which there is a subset $Q_{0}$ of $10^{10} n_{1}$ points of $Q$ so that all points of $Q_{0}$ are in the annulus $\left\{y \in R^{2}: r_{0} \leq d i s t\left(u_{0}, y\right) \leq r_{0}+3 r_{1}\right\}$ and are within the $5^{\circ}$ angular region formed by the rays (Fig. 1).

Using rays emanating from $u_{0}$, partition the region containing $Q_{0}$ into $10^{8}$ regions each covering at least $90 n_{1}$ points from $Q_{0}$. Denote the subsets by $\boldsymbol{Q}_{1}, \boldsymbol{Q}_{2}, \ldots, \boldsymbol{Q}_{10^{8}}$ and the angles determining them as $\theta_{1}, \theta_{2}, \ldots, \theta_{10^{8}}$. Let $\theta_{0}=\min \left\{\theta_{i}: 1 \leq i \leq 10^{8}\right\}$. Without loss of generality, there is some $i_{0}$ with $1 \leq i_{0} \leq 10^{8} / 2$ for which $\theta_{0}=\theta_{i_{0}}$. Relabel $Q_{i_{0}}$ as $Q_{1}^{\prime}$ and $Q_{10^{8}}$ as $Q_{2}^{\prime}$. Note that the angle $\theta$ in Fig. 2 is at least $10^{7} \theta_{0}$.

Now the boundary of the region containing $Q_{1}^{\prime}$ is very nearly a rectangle of width $3 r_{1}$ and height $\theta_{0} r_{0}$. We claim $\theta_{0} r_{0} \geq r_{1}$. Suppose not. Then the region containing $\boldsymbol{Q}_{1}^{\prime}$ has area at most $4 r_{1}^{2}$ and covers $90 n_{1}$ points of $\boldsymbol{Q}$. Furthermore, if $r_{1}>\theta_{0} r_{0}$, this region can be covered by eight discs of radius $r_{1}$ with centers at the points indicated in Fig. 3. This implies that there exists a disc of radius less than $r_{1}$ covering $n_{1}$ points of $Q$. The contradiction shows $\theta_{0} r_{0} \geq r_{1}$, as claimed.

Now that we have some control on the relative sizes of the three regions and

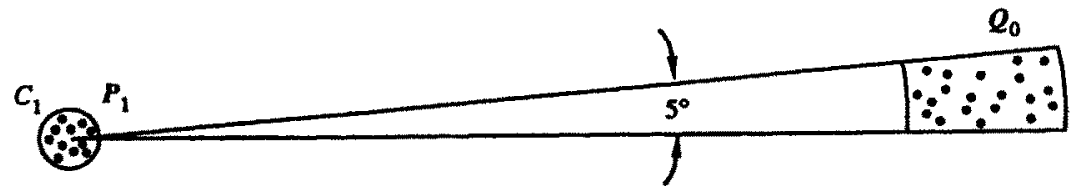

Fig. 1 




Fig. 2

the separation between them, it is easy to use the pigeon-hole principle to find a number $z \leq \theta_{0} r_{0}$ and rectangles $R_{0}, R_{1}$, as shown in Fig. 4, with $R_{0}$ covering a subset $\boldsymbol{P}_{2}$ of $n_{2}$ points from $\boldsymbol{P}_{1}$, while $R_{1}$ covers a subset $\boldsymbol{Q}_{1}^{\prime \prime}$ of $n_{2}$ points from $\boldsymbol{Q}_{1}^{\prime}$. Recall that $n_{2}=\left\lceil n /(\log n)^{20}\right\rceil<n_{1}$. For convenience, we change scale so that the distance (along line $L$ ) from the lower right corner of $R_{0}$ to the upper left corner of $R_{1}$ is exactly 1 . Then let $Q_{2}^{\prime \prime}$ be any subset of $n_{2}$ points from $Q_{2}^{\prime}$. Note that if $x \in P_{2}, y_{1} \in Q_{1}^{\prime \prime}$, and $y_{2} \in Q_{2}^{\prime \prime}$, then the angle formed by the segments $x y_{1}$ and $x y_{2}$ is at least $10^{6} \mathrm{z}$.

At this point we want to apply the covering lemma (see [5]-[7]). First extend the two vertical sides of rectangle $R_{0}$ upward to form a rectangle $R_{0}^{\prime}$ of width $z / 10$ and height 0.01 . Then choose a family $\mathscr{R}$ of subrectangles of $R_{0}^{\prime}$ so that:

(1) The sides of each rectangle $R \in \mathscr{R}$ are parallel to those of $R_{0}^{\prime}$.

(2) The interiors of the rectangles in $\mathscr{R}$ are pairwise disjoint.

(3) The ratio $w / h$ of width divided by height is $10 z$ for every rectangle in $\mathscr{R}$.

(4) Each rectangle in $\mathscr{R}$ contains at least $n_{2}^{3 / 5}$ and at most $1000 n_{2}^{3 / 5}$ points of $\boldsymbol{P}$.

(5) There are at least $n_{2}^{2 / 5} / 10^{6}$ rectangles in $\mathscr{R}$.

For a rectangle $R$, let $\boldsymbol{P}_{2}(R)$ denote those points of $\boldsymbol{P}_{2}$ covered by $R$. When $\boldsymbol{x} \in \boldsymbol{P}_{2}$ and $\boldsymbol{y} \in \boldsymbol{Q}_{1}^{\prime \prime} \cup \boldsymbol{Q}_{2}^{\prime \prime}$, we let $C(\boldsymbol{x}, \boldsymbol{y})$ denote the circle centered at $\boldsymbol{y}$ and passing through $x$. Now let $R \in \mathscr{R}, x \in \boldsymbol{P}_{2}(R)$, and $y \in Q_{1}^{\prime \prime}$. We claim that the circle $C(x, y)$ cannot cross both vertical sides of $R$ (Fig. 5).

To see that this claim is valid, let $R$ have height $h$ and width $w=10 z h$. Now the point $\boldsymbol{x}$ is covered by $R_{0}$, so $\boldsymbol{x}$ is at most $z$ above the line $L$. It is an easy exercise to show that in moving laterally an amount $w$, the circle $C(x, y)$ must rise at least $w / 5 z$ and this quantity exceeds $h$. This shows that $C(\boldsymbol{x}, y)$ cannot cross



Fig. 3 


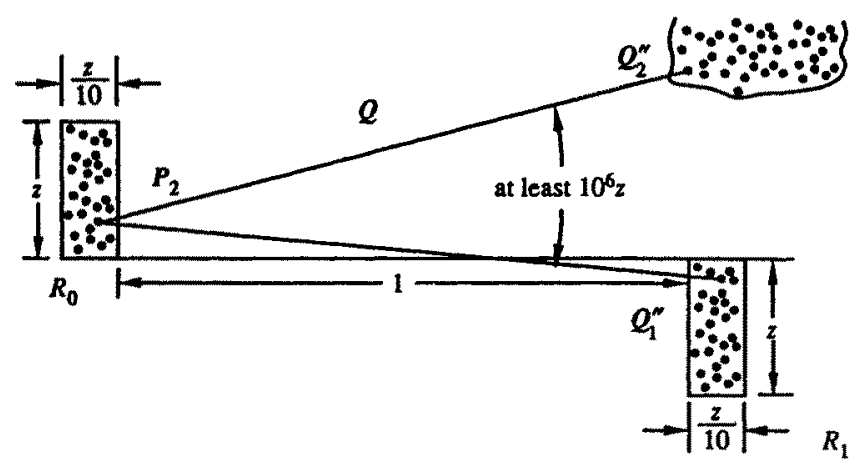

Fig. 4

both vertical sides of $R$. However, it is indeed possible for $C(x, y)$ to cross both the top and bottom sides of $R$.

Now let $R \in \mathscr{R}, x \in \boldsymbol{P}_{2}(R), \boldsymbol{y}_{1} \in \boldsymbol{Q}_{1}^{\prime \prime}$, and $\boldsymbol{y}_{2} \in \boldsymbol{Q}_{2}^{\prime \prime}$. We claim that it cannot happen that $C\left(x, y_{1}\right)$ and $C\left(x, y_{2}\right)$ both cross the top and bottom sides of $R$ (Fig. 6).

To see that this claim holds, we consider the angle formed by the line segments $x y_{1}$ and $x y_{2}$. In view of the relative separation between $Q_{1}^{\prime \prime}$ and $Q_{2}^{\prime \prime}$, this angle is at least $10^{6} z$. However, if both $C\left(x, y_{1}\right)$ and $C\left(x, y_{2}\right)$ cross the top and the bottom of $R$, then this angle is at most $100 z$. The contradiction completes the proof of the claim.

The Crossing Property. Let $R \in \mathscr{R}$. Subdivide $R$ into four subrectangles of equal size by taking the perpendicular bisectors of the sides of $R$. Let $R^{\prime}$ be any one of

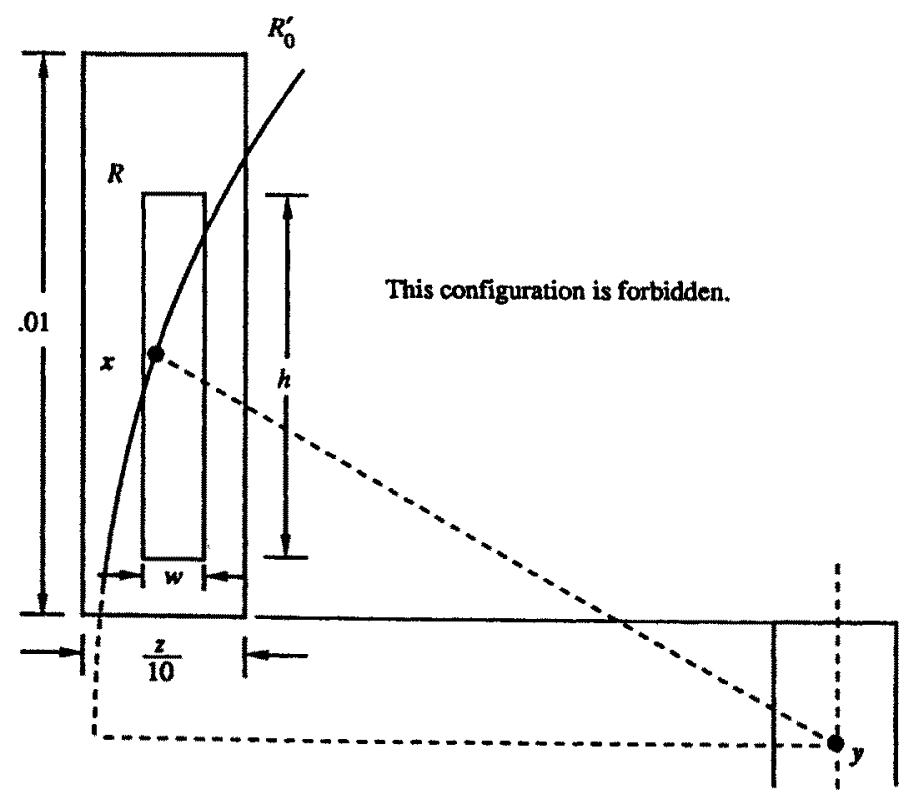

Fig. 5 


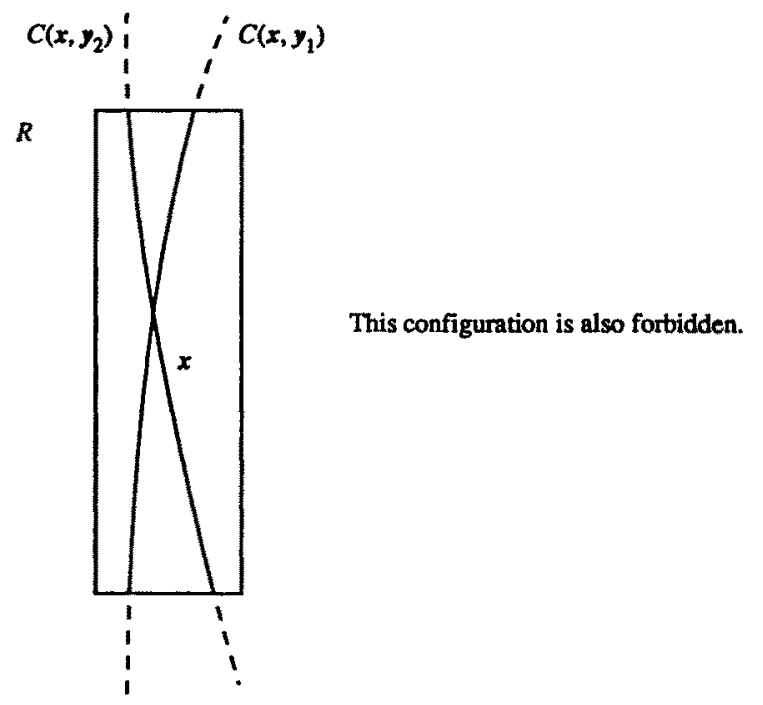

Fig. 6

these subrectangles and let $x, x^{\prime} \in P_{2}\left(R^{\prime}\right)$. Then, for every $y_{1} \in Q_{1}^{\prime \prime}$ and for every $y_{2} \in Q_{2}^{\prime \prime}$, either $C\left(x, y_{1}\right)$ and $C\left(x^{\prime}, y_{2}\right)$ cross at a point inside $R$, or $C\left(x^{\prime}, y_{1}\right)$ and $C\left(x, y_{2}\right)$ cross at a point inside $R$.

We comment that the proof of the Crossing Property is elementary and proceeds by a case-by-case analysis of the four subrectangles and the relative positions of $\boldsymbol{x}$ and $\boldsymbol{x}^{\prime}$ within them. However, we do note that the desired crossing may occur inside $R$ but not inside $R^{\prime}$ as illustrated in Fig. 7 .

The reason for introducing the Crossing Property is that we will force pairs of circles with centers in $\boldsymbol{Q}$ to cross at a point in the plane not occupied by a point of $\boldsymbol{P}$. We refer to this as a wasted crossing. The final contradiction is obtained by showing that the number of wasted crossings is larger than the number of pairs of circles from $\{C(\boldsymbol{x}, \boldsymbol{y}): \boldsymbol{x} \in \boldsymbol{P}, \boldsymbol{y} \in \boldsymbol{Q}\}$. Now set $i=1$. We describe a procedure which

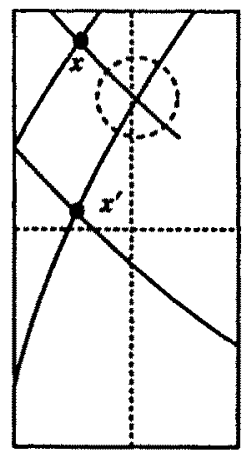

Fig. 7 
identifies particular points in $\boldsymbol{P}_{2} \cup \boldsymbol{Q}_{i}^{\prime \prime}$ and certain rectangles in $\mathscr{R}$. This argument will be repeated when $i=2$. Let $R \in \mathscr{R}, x \in R$, and $y \in Q_{i}^{\prime \prime}$. We call $(x, y)$ a rich pair if $C(x, y)$ contains at least two points of $P(R)$.

Lemma. The number of rich pairs is less than $n_{8}^{2}$.

Proof. Suppose the lemma is false and let $S$ be a set of $n_{8}^{2}$ rich pairs of the prescribed form. Choose a number $a \geq 2$ for which there are at least $n_{9}^{2}$ rich pairs $(x, y)$ for which $C(x, y)$ contains at least $a$ and at most $2 a$ points of $P(R)$ where $R$ is the rectangle containing $x$. Call such pairs uniform rich pairs. (Note that here we actually use the logarithmic factor.)

Then let

$$
\begin{gathered}
\mathscr{T}=\left\{\boldsymbol{x}, \boldsymbol{x}^{\prime}, y\right): \text { there exists a rectangle } R \in \mathscr{R} \text { containing both } \boldsymbol{x} \text { and } \boldsymbol{x}^{\prime}, \boldsymbol{y} \in \boldsymbol{Q}_{i}, \\
\left.\boldsymbol{x}^{\prime} \in C(\boldsymbol{x}, \boldsymbol{y}) \text { and both }(\boldsymbol{x}, \boldsymbol{y}) \text { and }\left(\boldsymbol{x}^{\prime}, y\right) \text { are uniform rich pairs }\right\} .
\end{gathered}
$$

Then $|\mathscr{T}| \geq n_{9}^{2} a$. For each $R \in \mathscr{R}$, let $\mathscr{T}(R)=\left\{\left(\boldsymbol{x}, \boldsymbol{x}^{\prime}, y\right) \in \mathscr{T}: x, x^{\prime} \in R\right\}$. Since $R$ contains at most $1000 n_{2}^{3 / 5}$ points of $\boldsymbol{P}$ and $\boldsymbol{Q}_{i}^{\prime \prime}$ contains $n_{2}$ points, there are at most $1000 n_{2}^{8 / 5}$ uniform rich pairs $(x, y)$ where $x \in R$ and $y \in Q_{i}^{\prime \prime}$. For each such pair, there are at most $2 a$ different $x^{\prime} \in R$ and for which $\left(x, x^{\prime}, y\right)$ is a triple in $\mathscr{T}(R)$. It follows that $|\mathscr{T}(R)| \leq 2000 n_{2}^{8 / 5} a<n_{2}^{8 / 5} a$. We also observe that $n_{2}^{2 / 5} / 10^{6} \leq|\mathscr{R}|<n_{1}^{2 / 5}$. Let $\mathscr{T}_{0}=\bigcup\left\{\mathscr{T}(R):|\mathscr{T}(R)|<n_{9}^{2} a / 2 n_{1}^{2 / 5}\right\}$. Then $\left|\mathscr{T}_{0}\right|<n_{9}^{2} a / 2$ and $\left|\mathscr{T}-\mathscr{T}_{0}\right| \geq n_{9}^{2} a / 2$. So there exists a number $b \geq n_{9}^{2} a / 2 n_{1}^{2 / 5}$ and a subfamily $\mathscr{R} \mathscr{R}^{\prime} \subseteq \mathscr{R}$ so that $b \leq|\mathscr{T}(R)| \leq 2 b$ for every $R \in \mathscr{R}^{\prime}$ and $\left|\bigcup\left\{\mathscr{T}(R): R \in \mathscr{R}^{\prime}\right\}\right| \geq n_{9}^{2} a / 10 \log n \geq n_{10}^{2} a$.

Let $\mathscr{T}_{1}=\bigcup\left\{\mathscr{T}(R): R \in \mathscr{R}^{\prime}\right\}$ and let $R \in \mathscr{R}^{\prime}$. For each distinct pair $\boldsymbol{x}, \boldsymbol{x}^{\prime} \in \boldsymbol{P}(R)$, let $\boldsymbol{T}\left(\boldsymbol{x}, \boldsymbol{x}^{\prime}\right)=\left\{y \in Q_{1}:\left(x, x^{\prime}, y\right) \in \mathscr{T}_{1}\right\}$. For each $R \in \mathscr{R}^{\prime}$, let

$$
\mathscr{T}_{2}(R)=\left\{\left(x, x^{\prime}, y\right) \in \mathscr{T}(R):\left|T\left(x, x^{\prime}\right)\right|<b /\left(2 \cdot 10^{6} n_{2}^{6 / 5}\right)\right\} .
$$

Since $|R| \leq 10^{3} n_{2}^{3 / 5}$, we see that $\left|\mathscr{T}_{2}(R)\right|<b / 2$. Set $\mathscr{T}_{3}(R)=\mathscr{T}(R)-\mathscr{T}_{2}(R)$. Since $\mathscr{T}(R) \geq b$, we see that $\left|\mathscr{T}_{3}(R)\right| \geq b / 2$. Then set $\mathscr{T}_{3}=\bigcup\left\{\mathscr{T}_{3}(R): R \in \mathscr{R}^{\prime}\right\}$, and observe that $\left|\mathscr{T}_{3}\right| \geq\left|\mathscr{T}_{1}\right| / 4 \geq n_{10}^{2} a / 4$.

When $\boldsymbol{x}$ and $\boldsymbol{x}^{\prime}$ are distinct points in $\boldsymbol{P}$, we call $\left(\boldsymbol{x}, \boldsymbol{x}^{\prime}\right)$ a standard pair if there is some $R \in \mathscr{R}^{\prime}$ for which $\boldsymbol{x}, \boldsymbol{x}^{\prime} \in \boldsymbol{P}(R)$ and $\left|T\left(\boldsymbol{x}, \boldsymbol{x}^{\prime}\right)\right| \geq b /\left(2 \cdot 10^{6} n_{2}^{6 / 5}\right)$. When $\left(\boldsymbol{x}, \boldsymbol{x}^{\prime}\right)$ is a standard pair, we let $L\left(x, x^{\prime}\right)$ denote the perpendicular bisector of the line segment passing through $\boldsymbol{x}$ and $\boldsymbol{x}^{\prime}$, and we let $\mathscr{L}$ denote the set of all perpendicular bisectors of standard pairs. Note that each line $L\left(x, x^{\prime}\right) \in \mathscr{L}$ contains at least $b /\left(2 \cdot 10^{6} n_{2}^{6 / 5}\right)$ points from the set $Q_{1}^{\prime \prime}$ (Fig. 8).

For each line $L \in \mathscr{L}$, let $|L|$ denote the number of points of $Q_{1}^{\prime \prime}$ which lie on $L$. As noted previously, $|L| \geq b /\left(2 \cdot 10^{6} n_{2}^{6 / 5}\right)$, but it is possible for $|L|$ to be much larger. Choose a value $e \geq b /\left(2 \cdot 10^{6} n_{2}^{6 / 5}\right)$ and a subset $\mathscr{L}_{0} \in \mathscr{L}$ so that

(1) $e \leq|L| \leq 2 e$ for every $L \in \mathscr{L}_{0}$;

(2) there are at least $n_{11}^{2} a$ triples in $\mathscr{T}_{3}$ of the form $\left(x, x^{\prime}, y\right)$ where $\left(x, x^{\prime}\right)$ is a standard pair; and

(3) $L\left(x, x^{\prime}\right) \in \mathscr{L}_{0}$. 


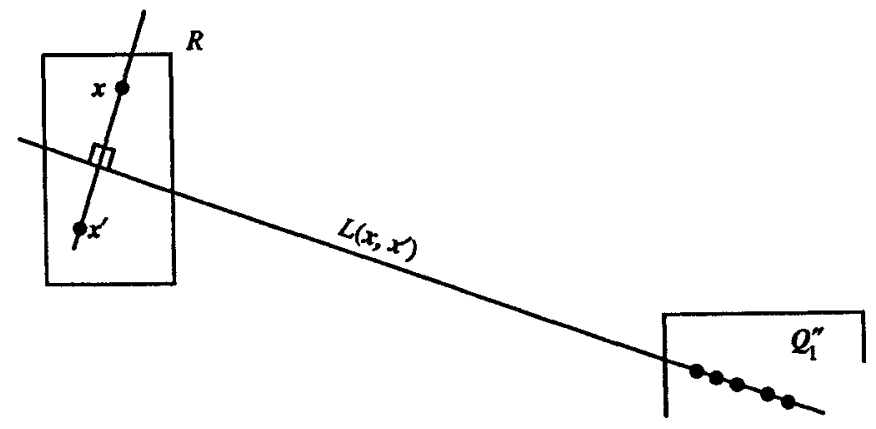

Fig. 8

Case 1: $e \geq 10 \sqrt{n}$. Since $\left|Q_{1}^{\prime \prime}\right|<n$, a simple counting argument shows $\left|\mathscr{L}_{0}\right| \leq 100 n / e$. Let $d=n^{4 / 5} /(\log n)^{c}$. For each line $L \in \mathscr{L}_{0}$, we know that $L$ contains at most $2 e$ points of $Q_{1}$. For each $y \in Q_{1}$ which lies on $L$, there are at most $2 a d$ standard pairs $\left(\boldsymbol{x}, \boldsymbol{x}^{\prime}\right)$ for which $L=L\left(\boldsymbol{x}, \boldsymbol{x}^{\prime}\right)$ and $\left(\boldsymbol{x}, \boldsymbol{x}^{\prime}, \boldsymbol{y}\right) \in \mathscr{T}$. It follows that

$$
n_{11}^{2} a \leq \frac{100 n}{e} 2 e \cdot 2 a d<\frac{10^{3} n^{9 / 5} a}{(\log n)^{c}}
$$

which is clearly false.

Case 2: $e<10 \sqrt{n}$. In this case we use the inequality in Theorem 2 to bound $\left|\mathscr{L}_{\mathrm{o}}\right|$. Note that $e \geq 2$, and even if $e>\sqrt{n}$, we can certainly say $\left|\mathscr{L}_{0}\right|<10^{200} n^{2} / e^{3}$. It follows that

$$
n_{11}^{2} a \leq \frac{10^{200} n^{2}}{e^{3}} \cdot 2 e \cdot 2 a d
$$

Thus $e \leq 10^{101} n^{7 / 5} / n_{11}(\log n)^{c / 2}$. However, we already know $e \geq b /\left(2 \cdot 10^{6} n_{2}^{6 / 5}\right) \geq$ $n_{9}^{2} a /\left(4 \cdot 10^{6} \cdot n_{2}^{6 / 5} \cdot n_{1}^{2 / 5}\right)$. Since $a \leq 2$, these inequalities for $e$ can be combined to show $n_{12}^{3}(\log n)^{c / 2} \leq 10^{110} n^{3}$, which is false. The contradiction completes the proof of the lemma.

Again set $i=1$. We next describe a selection process which identifies particular points and rectangles. This process will also be repeated when $i=2$. Let $R \in \mathscr{R}$, $\boldsymbol{x} \in \boldsymbol{P}_{\mathbf{2}}(R)$, and $\boldsymbol{y} \in \boldsymbol{Q}_{i}^{\prime \prime}$. We call a pair $(\boldsymbol{x}, \boldsymbol{y})$ a lean pair if $C(\boldsymbol{x}, \boldsymbol{y})$ contains no other point of $\boldsymbol{P}(R)$ besides $\boldsymbol{x}$. In view of the lemma, we may choose a set $W$ of $n_{3}^{2}$ lean pairs.

Let $R \in \mathscr{R}$ and let $\boldsymbol{x} \in \boldsymbol{P}(R)$. We say that $\boldsymbol{x}$ is normal if there are at least $n_{\mathbf{4}}$ points in the set $N_{i}(\boldsymbol{x})=\left\{\boldsymbol{y} \in \boldsymbol{Q}_{i}^{\prime \prime}:(\boldsymbol{x}, \boldsymbol{y})\right.$ is lean $\}$. Call a rectangle $R \in \mathscr{R}$ normal if it contains at least $n_{5}^{3 / 5}$ normal points. So we may choose a set $\mathscr{R}_{0}$ of $n_{6}^{2 / 5}$ normal rectangles. 
From each $R \in \mathscr{R}_{0}$, we may select a set $S_{0}(R)$ of $n_{5}^{3 / 5}$ normal points. For each $x \in S_{0}(R)$, we may choose a set $N_{i}^{\prime}(x)$ of $n_{4}$ points from $N_{i}(x)$.

Consider the set $\boldsymbol{G}=\bigcup\left\{S_{0}(R): R \in \mathscr{R}_{0}\right\}$. Then $|\boldsymbol{G}|=n_{6}^{2 / 5} n_{5}^{3 / 5}>n_{6}$. Now we repeat the argument in our lemma when $i=2$ but consider only the pairs of the form $(x, y)$ where $x \in G$ and $y \in Q_{2}$. We conclude that there exists a set $\mathscr{R}_{1}$ of $n_{7}^{2 / 5}$ rectangles from $\mathscr{R}_{0}$ so that:

For every rectangle $R \in \mathscr{R}_{1}$, there is a set $S_{1}(R)$ of $n_{7}^{3 / 5}$ points covered by $R$ so that:

(1) For each $x \in S_{1}(R)$, there are sets $N_{1}^{\prime \prime}(x) \subseteq Q_{2}^{\prime \prime}$ and $N_{2}^{\prime \prime}(x) \subseteq Q_{2}^{\prime \prime}$ so that $\left|N_{1}^{\prime \prime}(x)\right|=\left|N_{2}^{\prime \prime}(x)\right|=n_{7}$.

(2) For each $x \in S_{1}(R)$ and each $i=1,2$, and for every $y \in N_{i}^{\prime \prime}(x),(x, y)$ is a lean pair.

We now count wasted crossings. It is clear that the number of wasted crossings cannot exceed $n^{2} d^{2}$. However, there are $n_{7}^{2 / 5}$ rectangles in $\mathscr{R}_{1}$. In the appropriate subrectangle, there are at least $n_{8}^{3 / 5}$ points, so at least $n_{9}^{6 / 5}$ pairs of points. For each pair, there are at least $n_{7}^{2}$ wasted crossings. This requires $n_{7}^{2 / 5} n_{9}^{6 / 5} n_{7}^{2} \leq n^{2} d^{2}$ which is false. The contradiction completes the proof of Theorem 3.

\section{Concluding Remarks}

It is probably possible to modify the arguments in this paper to show that $d(n) \geq n^{4 / 5} / \omega(n)$ where $\omega(n) \rightarrow \infty$ arbitrarily slowly and $n$ is sufficiently large. In fact, perhaps it can be shown that $d(n) \geq \varepsilon n^{4 / 5}$ for some absolute constant $\varepsilon>0$. We find little reason to attempt such improvements since they would still leave us far from the conjectured lower bound which we suspect is correct.

Finally, we comment that the argument presented in this paper does not show that there is some point from which there are at least $n^{4 / 5} /(\log n)^{c}$ different distances.

\section{Acknowledgment}

The authors would like to express their sincere appreciation to János Pach for a detailed reading of a preliminary version of this manuscript and for many helpful suggestions and corrections.

\section{References}

1. J. Beck, Different Distances, preprint.

2. F. R. K. Chung, On the Number of Different Distances Determined by $n$ Points in the Plane, $J$. Combin. Theory Ser. A 36 (1984), 342-354.

3. P. Erdös, On Sets of Distances of $n$ Points, Amer. Math. Monthly 53 (1946), 248-250.

4. L. Moser, On the Different Distances Determined by $n$ Points, Amer. Math. Monthly 59 (1952), 85-91. 
5. J. Spencer, E. Szemerédi, and W. Trotter, Unit Distances in the Euclidean Plane, in Graph Theory and Combinatorics (B. Bollabás, ed.), Academic Press, New York, 1984, pp. 293-303.

6. E. Szemerédi and W. Trotter, A Combinatorial Distinction between the Euclidean and Projective Planes, European I. Combin. 4 (1983), 385-394.

7. E. Szemerédi and W. Trotter, Extremal Problems in Discrete Geometry, Combinatorica 3 (1983), $381-392$.

Received June 23, 1989, and in revised form February 20, 1991. 